\title{
Baujardia mirabilis gen. n., sp. n. from pitcher plants and its phylogenetic position within Panagrolaimidae (Nematoda: Rhabditida)
}

\author{
Wim Bert ${ }^{1, *}$, Irma TANDINGAN De LeY ${ }^{2}$, Rita VAN DRIESSChE ${ }^{1}$, \\ Hendrik SEGERS ${ }^{3}$ and Paul DE LEY ${ }^{2}$ \\ ${ }^{1}$ Department of Biology, Ghent University, Ledeganckstraat35, 9000 Gent, Belgium \\ ${ }^{2}$ Department of Nematology, University of California, Riverside, CA 92521, USA \\ ${ }^{3}$ Freshwater Laboratory, Royal Belgium Institute for Natural Sciences, Vautierstraat 29, 1000 Brussels, Belgium
}

Received: 16 September 2002; revised: 13 January 2003

Accepted for publication: 13 January 2003

\begin{abstract}
Summary - Measurements, line drawings and scanning electromicrographs are provided of Baujardia mirabilis gen. n., sp. n., isolated from pitcher fluid of Nepenthes mirabilis from Thailand. The new genus differs from all known nematodes in having two opposing and offset spermatheca-like pouches at the junction of oviduct and uterus. It also differs from most known Rhabditida in having four cephalic setae instead of papillae. Phylogenetic analysis of small subunit rDNA sequence data robustly places the new genus within Panagrolaimidae as a sister taxon to Panagrellus. These unusual nematodes resemble Panagrellus in body size (1.8-2.7 $\mathrm{mm}$ in females, 1.3-1.9 $\mathrm{mm}$ in males) and in the monodelphic, prodelphic female reproductive system with thickened vaginal walls and prominent postvulval sac. However, they differ from Panagrellus in the characters mentioned above, in their comparatively longer stegostom and in the shape of the male spicules. Because of its aberrant characters, inclusion of this new genus in Panagrolaimidae requires changes to the family diagnosis.
\end{abstract}

Keywords - Nepenthes, new genus, new species, Panagrellus, phylogeny, taxonomy, Thailand.

During a visit to Tung-Kai Botanical Garden in Trang Province, near the Isthmus of Kra, Southern Thailand, one of us (Hendrik Segers) sampled the liquid contained within pitchers of the carnivorous plant Nepenthes mirabilis (Lour.) Druce, in order to obtain some of the peculiar rotifers recorded from such habitats. Except for some unidentifiable bdelloids, no rotifers were found, but the samples contained abundant material of a remarkable new nematode genus together with some diplogastrids. Some of the unique morphological characters of this new genus make its placement difficult. In order to obtain additional information about the identity of the nematode and its position in relation to known taxa, we conducted molecular phylogenetic analyses based on the small subunit (SSU) ribosomal DNA. In this paper, we describe this new genus and species from Nepenthes and present an emended diagnosis of the family Panagrolaimidae.

\section{Materials and methods}

\section{SAMPLING}

Samples were collected by filtering the content of some 50 pitchers through a $50 \mu \mathrm{m}$ plankton net and were immediately fixed by adding one tenth of the sample volume of concentrated formalin (36\%) at ambient temperature. Nepenthes mirabilis was encountered near the end of the trail through the primary swamp forest of Tung Kai Botanical garden. A single bulk sample was collected on 18 July 1999. Several additional samples, including a sample preserved in $70 \%$ ethanol, were collected on 13 January 2002.

\section{MORPHOLOGICAL OBSERVATIONS}

For light microscopy, nematodes were processed to anhydrous glycerol (Seinhorst, 1959) and mounted on aluminium slides with double cover slips. Measurements and

*Corresponding author, e-mail: wim.bert@ rug.ac.be 
illustrations were prepared from camera lucida line drawings using Olympus BX50 and BX51 DIC microscopes and the drawings were prepared using Illustrator ${ }^{\circledR} 8.0$ software (Adobe Systems, Mountain View, CA, USA). For scanning electron microscopic (SEM) observation, glycerine-embedded nematodes were first transferred into a drop of glycerine, gradually transferred to distilled water over a period of $4 \mathrm{~h}$, then dehydrated in a $25,50,75$, 95 , and $100 \%$ ethanol series at $2 \mathrm{~h}$ intervals, followed by an overnight dehydration in $100 \%$ ethanol. Dehydrated nematodes were critical-point dried with $\mathrm{CO}_{2}$ (Wergin, 1981), sputter coated with $20 \mathrm{~nm}$ gold, and examined with a JEOL JSM-840 at $15 \mathrm{kV}$.

In addition to slide voucher specimens, morphology of the new genus was recorded as video clips that mimic multifocal observation through a light microscope following the Video Capture and Editing procedures developed by De Ley and Bert (2002). The resulting virtual specimens are available on the web (http://faculty.ucr.edu/ pdeley/vce/Nep/nep.html).

Terminology on stoma morphology was adapted from De Ley et al. (1995). The papilla formula follows De Ley et al. (1999): pre- and postcloacal papillae are separated by ' $\%$, with precloacal papillae listed first, and the number of pairs in each cluster of papillae separated from the adjacent cluster with a '+'. The 'p' for phasmid pair is omitted, as we did not observe any phasmids.

\section{MOLECULAR ANALYSIS}

The nematodes were rehydrated in distilled water for $1 \mathrm{~h}$ to remove ethanol. DNA extraction and sequencing were as described in Tandingan De Ley et al. (2002).

The DNA sequence of Baujardia gen. n. (GenBank accession number: AF547385) was aligned with the published sequences of 21 other taxa. A secondary structure alignment was constructed using Dedicated Comparative Sequence Editor (DCSE v.3.4) (De Rijk \& De Wachter, 1993), based on the unweighted SSU rRNA model of Van de Peer et al. (1998) derived from base-pairings detected as compensating mutations, with equal weighting to stems and loops. Using Modeltest (Posada \& Crandall, 1988) this alignment was analysed to determine the appropriate model of DNA substitution (GTR $+\mathrm{I}+\Gamma, 6$ substitution types) and to estimate the substitution rates, relative base frequencies, gamma distribution shape parameter, and proportion of invariable sites.

Using the parameter estimates obtained from Modeltest, molecular phylogenetic relationships were reconstructed with neighbour joining (NJ), maximum parsi- mony (MP) and maximum likelihood (ML) algorithms implemented in PAUP* 4b10 (Swofford, 2002). During the analyses, trees were rooted using Plectus. Gaps were always treated as missing data. To estimate overall support for monophyly, NJ distance calculations were performed with 3000 bootstrap replications with corrections for multiple substitutions using Log Determinant distance measure (Lockhart et al., 1994). MP heuristic analysis was performed with 3000 bootstrap replicates and without bootstrapping with 100 replicates of random branch addition. Finally, a non-bootstrapheuristic ML search was also performed with 100 replicates of random branch addition. The trees were then compared statistically using the Kishino and Hasegawa (1989) pairwise tests with ML and MP optimality criteria, and Templeton test with MP optimality criterion. Cladograms were examined with TREEVIEW 1.6 (Page, 1996) and converted into graphic files for Adobe Illustrator ${ }^{\circledR} 9.0$.

\section{Baujardia mirabilis* gen. n., sp. n.}

(Figs 1-4)

\section{MEASUREMENTS}

See Table 1.

\section{DESCRIPTION}

\section{Female}

Body large (1.8-2.7 mm), almost straight after fixation. Cuticle finely and faintly annulated, $0.8 \mu \mathrm{m}$ wide at midbody. Lateral field narrow $(2.5 \mu \mathrm{m}$ wide at midbody), starting at end of procorpus and becoming inconspicuous at anal region. At midbody, lateral field forming a single protruding ridge flanked by fine, faint, indistinct longitudinal lines. Mouth opening partly covered by six liplets each bearing a labial papilla. Liplets continuous with six discrete lips separated by shallow grooves. Four short cephalic setae, 3-4 $\mu \mathrm{m}$ long, placed at base of subventral and subdorsal lips. Amphid opening slit like, encircled by a round shallow incisure and situated two discontinuous annuli posterior to setae. Stoma triradiate, relatively wide along full length (on average one third as wide as head diam.). Lengths of cheilostom, gymnostom

\footnotetext{
* The genus name is given in honor of Dr Pierre Baujard for his outstanding contributions to nematology. It is to be regarded as feminine in gender. The specific epithet refers to the biotope this nematode inhabits, i.e., the pitcher fluid of Nepenthes mirabilis.
} 

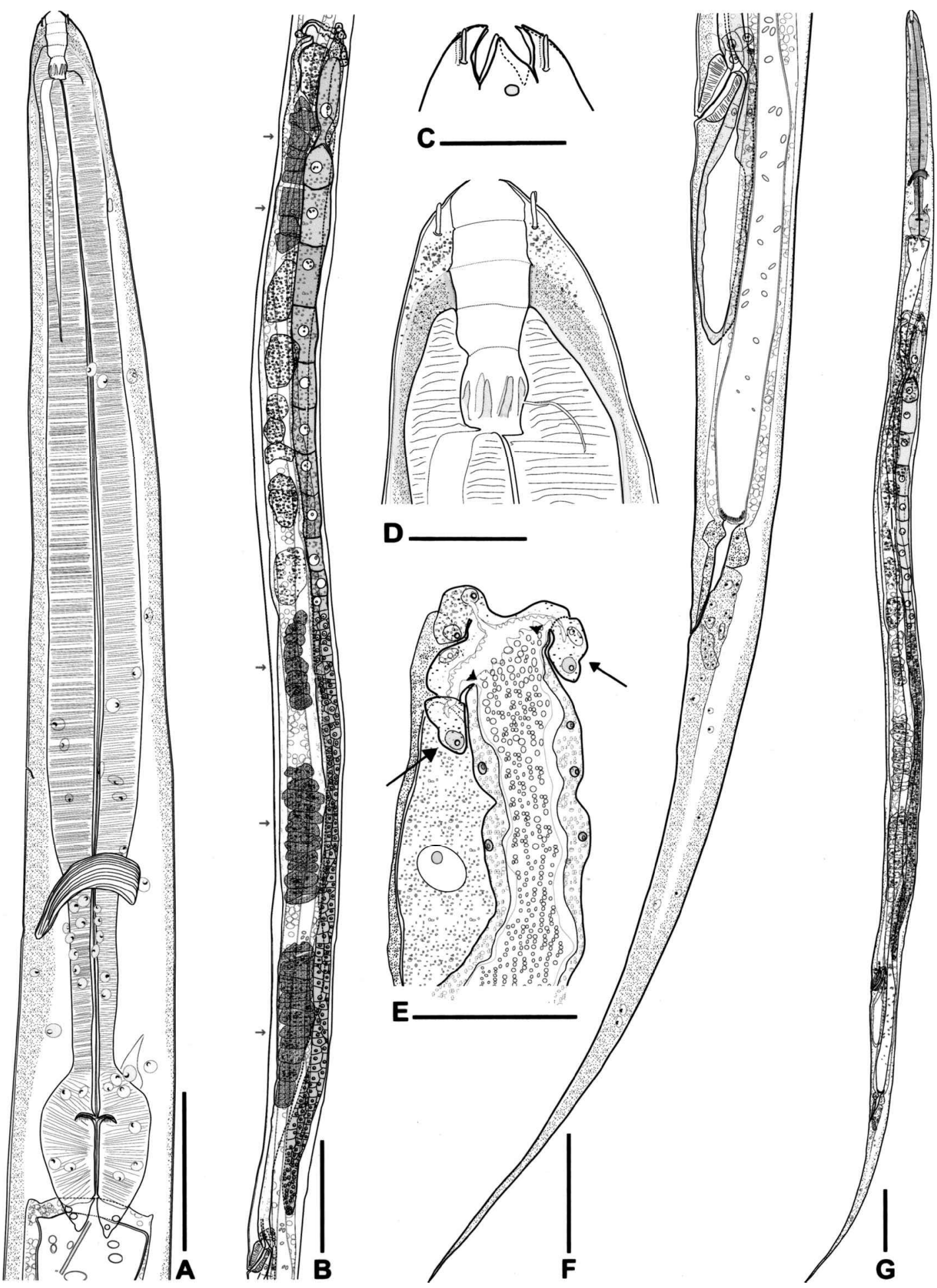

Fig. 1. Baujardia mirabilis gen. $n$., sp. n., female (holotype). A: Neck region; B: Reproductive system showing granulated sperm cells (arrows); C: Head region (superficial view); D: Stoma; E: End of ovary; oviduct, spermatheca with two offset pouches (arrows) and beginning of uterus (arrowheads); F: Vulva-tail region; G: Entire female. (Scale bars: B, $G=100 \mu \mathrm{m} ; A, F=50 \mu \mathrm{m} ; C, D, E=$ $10 \mu \mathrm{m}$. 

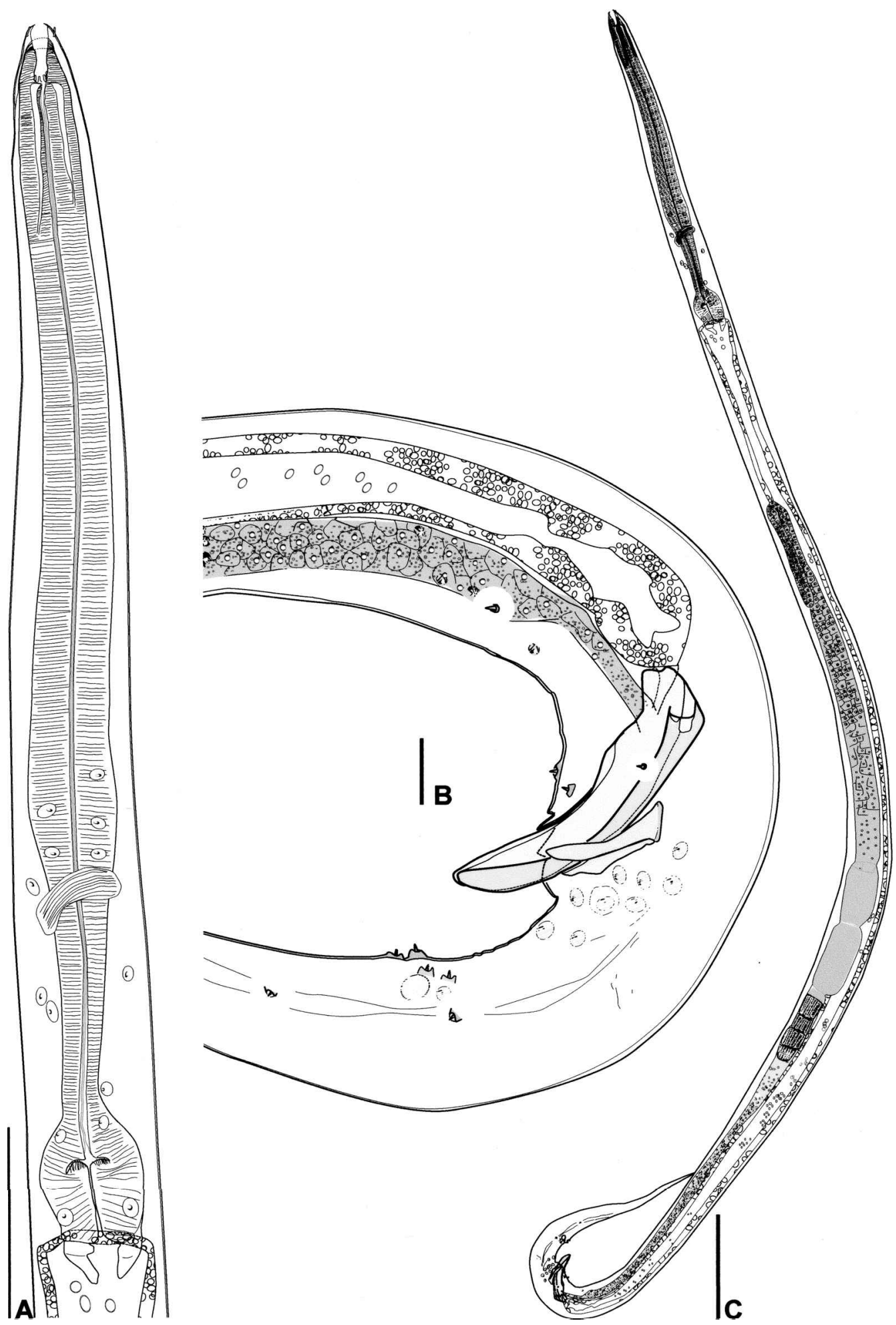

Fig. 2. Baujardia mirabilis gen. n., sp. n., male. A: Neck region; B: Cloacal region with copulatory papillae; C: Entire male. (Scale bars: $C=100 \mu \mathrm{m} ; A=50 \mu \mathrm{m} ; B=10 \mu \mathrm{m}$.) 

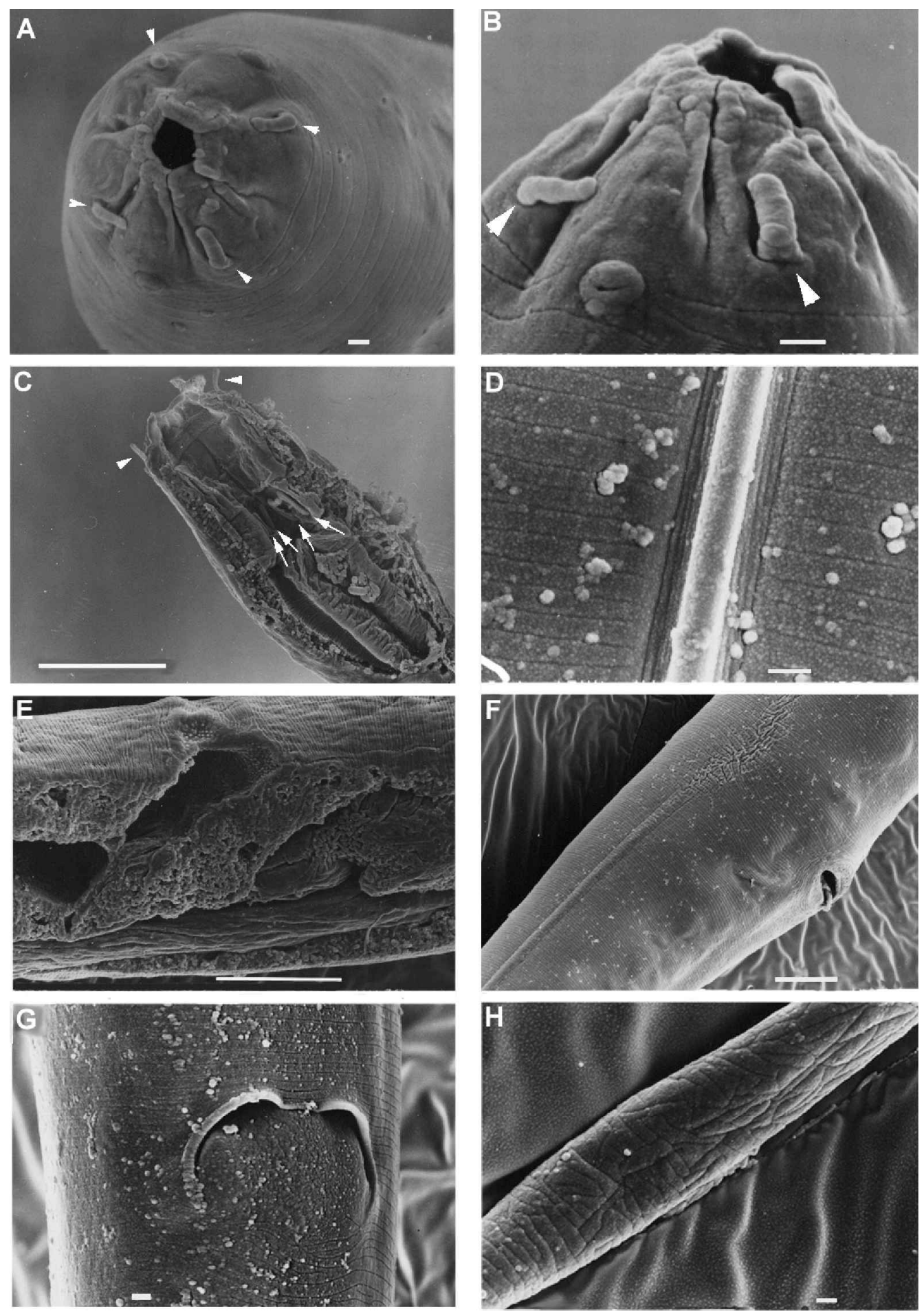

Fig. 3. Baujardia mirabilis gen. n., sp. n., SEM studies of female. A: En face view; B: Lateral view head; C: Longitudinal section of stoma region; D: Lateral field (at middle of body); E: Longitudinal section at the vulval region; $F$ : Vulva lateral view; G: Anus ventral view; H: Middle of tail. Arrowheads: cephalic setae. Arrows: digitate stoma projections. (Scale bars: $C, E, F=10 \mu m ; A, B, D, G, H$ $=1 \mu \mathrm{m}$.) 

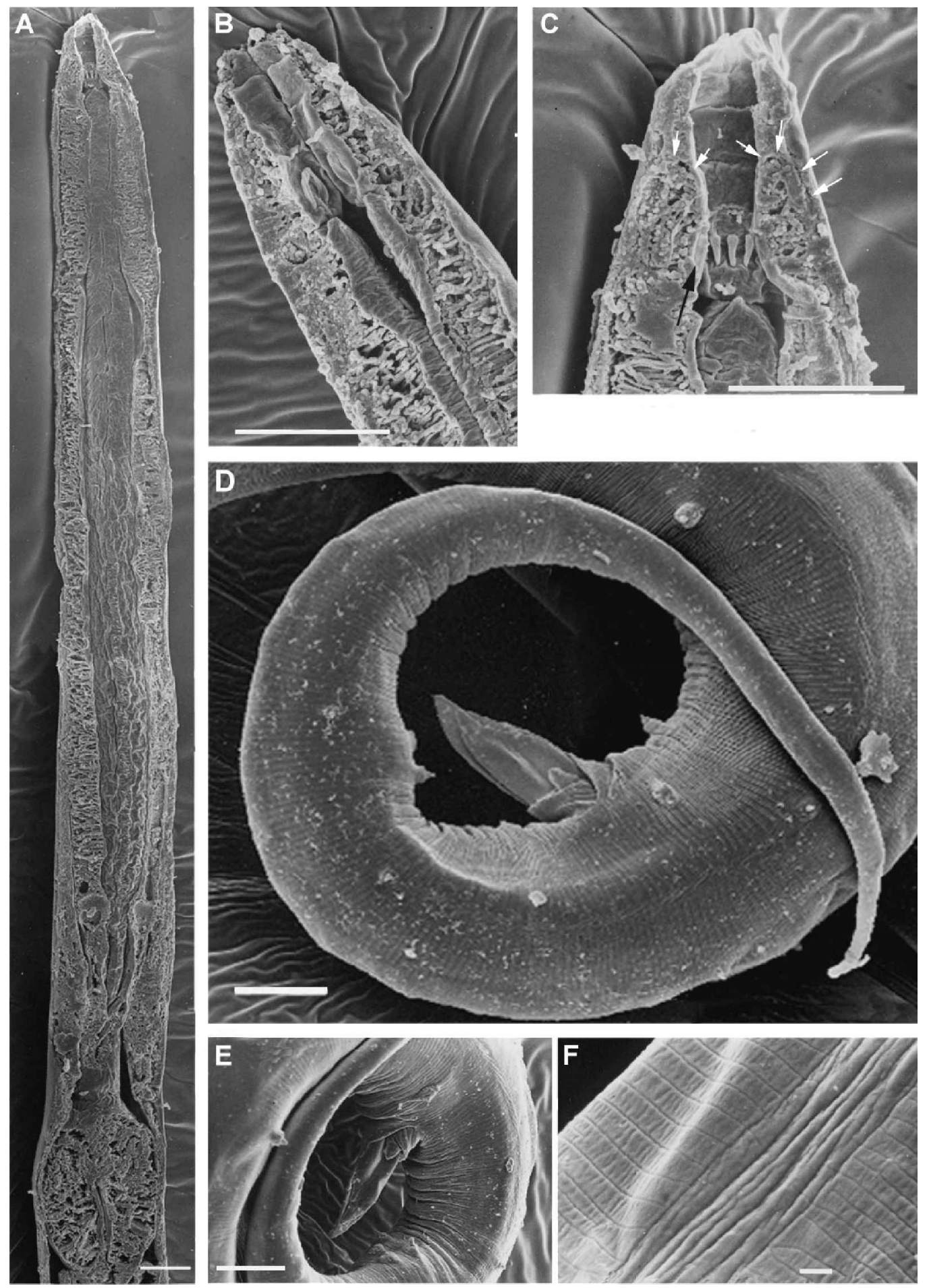

Fig. 4. Baujardia mirabilis gen. n., sp. n., SEM studies of male. A: Longitudinal section of neck region; B: Longitudinal section of stoma region showing the subventral sectors; $C$ : Longitudinal section of stoma region showing the dorsal sector, black arrow $=$ projection of subventral sector, white arrows = basal lamina; D: Cloacal and tail region, lateral view; E: Cloacal region, ventral view; F: Lateral field (at middle of body). (Scale bars: A, B, C, D, E =10 $\mu \mathrm{m} ; F=1 \mu \mathrm{m}$.) 
Table 1. Morphometric data of two populations of Baujardia mirabilis gen. $n .$, sp. $n$. Measurements are in $\mu m$ and presented as: mean \pm standard deviation (range).

\begin{tabular}{|c|c|c|c|c|c|c|c|}
\hline & \multicolumn{5}{|c|}{1999 Population (Type) } & \multicolumn{2}{|c|}{2002 Population ${ }^{*}$} \\
\hline & Holotype & $\begin{array}{c}\text { Female } \\
\text { paratypes }\end{array}$ & $\mathrm{CV}$ & $\begin{array}{c}\text { Male } \\
\text { paratypes }\end{array}$ & $\mathrm{CV}$ & $\begin{array}{c}\text { Female } \\
\text { paratypes }\end{array}$ & $\mathrm{CV}$ \\
\hline $\mathrm{n}$ & & 19 & & 17 & & 12 & \\
\hline $\mathrm{L}$ & 2138 & $\begin{array}{l}2316 \pm 238 \\
(1837-2745)\end{array}$ & 10.3 & $\begin{array}{c}1694 \pm 175 \\
(1350-1944)\end{array}$ & 10.3 & $\begin{array}{l}2113 \pm 380 \\
(1512-2550)\end{array}$ & 18 \\
\hline $\mathrm{a}$ & 34.7 & $\begin{array}{c}31 \pm 3.5 \\
(22-36)\end{array}$ & 11.3 & $\begin{array}{c}34.3 \pm 4.1 \\
(27-43)\end{array}$ & 11.8 & $\begin{array}{c}32.5 \pm 4.5 \\
(23-37)\end{array}$ & 14 \\
\hline $\mathrm{b}$ & 5.7 & $\begin{array}{l}6.2 \pm 0.5 \\
(5.3-7.4)\end{array}$ & 8.7 & $\begin{array}{l}5.2 \pm 0.5 \\
(4.1-5.9)\end{array}$ & 9.6 & $\begin{array}{l}6.3 \pm 1.2 \\
(4.4-7.7)\end{array}$ & 19 \\
\hline $\mathrm{c}$ & 7 & $\begin{array}{l}7.5 \pm 0.6 \\
(6.2-8.9)\end{array}$ & 8.4 & $\begin{array}{c}8.7 \pm 1.1 \\
(6.7-11)\end{array}$ & 12.4 & $\begin{array}{l}6.6 \pm 0.5 \\
(6.1-7.5)\end{array}$ & 7.2 \\
\hline$c^{\prime}$ & 10.5 & $\begin{array}{c}10.1 \pm 1 \\
(7.3-11.7)\end{array}$ & 10.1 & $\begin{array}{l}6.3 \pm 0.7 \\
(4.6-7.5)\end{array}$ & 10.8 & $\begin{array}{l}10.6 \pm 2.3 \\
(6.5-13.5)\end{array}$ & 21 \\
\hline Maximum body diam. & 61.6 & $\begin{array}{l}76 \pm 15 \\
(54-107)\end{array}$ & 19.8 & $\begin{array}{c}50 \pm 7.7 \\
(36-68)\end{array}$ & 15.5 & $\begin{array}{c}64.3 \pm 13.2 \\
(48-84)\end{array}$ & 21 \\
\hline Stoma length & 20 & $\begin{array}{c}20 \pm 0.8 \\
(18-22)\end{array}$ & 4.1 & $\begin{array}{l}18 \pm 1 \\
(16-20)\end{array}$ & 5.5 & $\begin{array}{c}19 \pm 1.7 \\
(17-22)\end{array}$ & 8.7 \\
\hline Head diam. (at amphid) & 13.3 & $\begin{array}{c}13 \pm 1.0 \\
(12-15)\end{array}$ & 7.8 & $\begin{array}{c}12 \pm 0.8 \\
(9-13)\end{array}$ & 7.1 & $\begin{array}{c}12 \pm 0.7 \\
(11-13)\end{array}$ & 6 \\
\hline Stoma length/head diam. & 1.5 & $\begin{array}{l}1.5 \pm 0.1 \\
(1.3-1.7)\end{array}$ & 6.9 & $\begin{array}{c}1.5 \pm 0.2 \\
(1.3-2)\end{array}$ & 10.4 & $\begin{array}{l}1.6 \pm 0.2 \\
(1.4-1.8)\end{array}$ & 9.7 \\
\hline Seta length & 4.1 & $\begin{array}{l}2.8 \pm 0.6 \\
(1.7-4.2)\end{array}$ & 23 & $\begin{array}{l}2.7 \pm 0.5 \\
(1.7-3.5)\end{array}$ & 17.2 & $\begin{array}{l}2.6 \pm 0.4 \\
\quad(2-3)\end{array}$ & 17 \\
\hline Amphid width & 6.7 & $\begin{array}{l}6 \pm 1.1 \\
(4-8.5)\end{array}$ & 19 & $\begin{array}{l}5.4 \pm 1.1 \\
(3-7)\end{array}$ & 19.5 & $\begin{array}{l}5.5 \pm 0.9 \\
(4-7)\end{array}$ & 17 \\
\hline Neck length & 375 & $\begin{array}{l}372 \pm 16 \\
(337-402)\end{array}$ & 4.4 & $\begin{array}{l}326 \pm 19 \\
(285-352)\end{array}$ & 5.8 & $\begin{array}{c}336 \pm 28.7 \\
(294-378)\end{array}$ & 8.5 \\
\hline Corpus length & 252 & $\begin{array}{c}253 \pm 16.3 \\
(222-280)\end{array}$ & 6.4 & $\begin{array}{l}216 \pm 15 \\
(186-237)\end{array}$ & 6.9 & $\begin{array}{l}219 \pm 19 \\
(189-250)\end{array}$ & 8.7 \\
\hline Procorpus length & 145 & $\begin{array}{c}135 \pm 11.7 \\
(117-153)\end{array}$ & 8.7 & $\begin{array}{c}117 \pm 8.6 \\
(97-127)\end{array}$ & 7.3 & $\begin{array}{c}118 \pm 11.6 \\
(108-132)\end{array}$ & 9.8 \\
\hline Metacorpus length & 107 & $\begin{array}{l}115 \pm 11 \\
(97-132)\end{array}$ & 9.5 & $\begin{array}{c}98.4 \pm 8.1 \\
(78-112)\end{array}$ & 8.2 & $\begin{array}{l}114 \pm 9.1 \\
(103-123)\end{array}$ & 7.9 \\
\hline Isthmus length & 60 & $\begin{array}{c}57 \pm 4.1 \\
(51-62)\end{array}$ & 7.1 & $\begin{array}{l}51.2 \pm 4.4 \\
(40-57)\end{array}$ & 8.5 & $\begin{array}{c}53.8 \pm 5.1 \\
(48-61)\end{array}$ & 9.5 \\
\hline Bulb length & 42.5 & $\begin{array}{c}46 \pm 3.4 \\
(40-52)\end{array}$ & 7.3 & $\begin{array}{c}43 \pm 5.3 \\
(35-52)\end{array}$ & 12.3 & $\begin{array}{c}40 \pm 3.8 \\
(35-46)\end{array}$ & 9.4 \\
\hline Corpus/isthmus & 4.2 & $\begin{array}{l}4.4 \pm 0.4 \\
(3.7-5.2)\end{array}$ & 8.7 & $\begin{array}{l}4.2 \pm 0.4 \\
(3.4-4.8)\end{array}$ & 8.6 & $\begin{array}{l}4.1 \pm 0.3 \\
(3.6-4.6)\end{array}$ & 8.3 \\
\hline Excretory pore position & 232 & $\begin{array}{l}224 \pm 22 \\
(180-260)\end{array}$ & 9.8 & $\begin{array}{c}199 \pm 27.3 \\
(155-252)\end{array}$ & 13.7 & $\begin{array}{c}220 \pm 25.5 \\
(186-252)\end{array}$ & 12 \\
\hline Nerve ring position & 270 & $\begin{array}{c}269 \pm 20.7 \\
(235-312)\end{array}$ & 7.7 & $\begin{array}{c}237 \pm 15.2 \\
(210-237)\end{array}$ & 6.4 & $\begin{array}{c}255 \pm 30.3 \\
(216-294)\end{array}$ & 12 \\
\hline EP (as \% Neck length) & 62 & $\begin{array}{c}59 \pm 4.2 \\
(53-66)\end{array}$ & 7.1 & $\begin{array}{c}61.5 \pm 7.1 \\
(48-73)\end{array}$ & 11.6 & $\begin{array}{c}66 \pm 5.6 \\
(57-73)\end{array}$ & 8.5 \\
\hline NR (as \% Neck length) & 72 & $\begin{array}{c}72.2 \pm 3.4 \\
(66-80)\end{array}$ & 4.8 & $\begin{array}{c}73 \pm 3.5 \\
(65-79)\end{array}$ & 4.8 & $\begin{array}{c}75.8 \pm 6.8 \\
(66-86)\end{array}$ & 8.9 \\
\hline Tail length & 305 & $\begin{array}{l}307 \pm 18 \\
(275-345)\end{array}$ & 6 & $\begin{array}{c}195 \pm 15.4 \\
(162-225)\end{array}$ & 7.9 & $\begin{array}{c}317 \pm 48.1 \\
(243-378)\end{array}$ & 15 \\
\hline
\end{tabular}


Table 1. (Continued).

\begin{tabular}{|c|c|c|c|c|c|c|c|}
\hline & \multicolumn{5}{|c|}{1999 Population (Type) } & \multicolumn{2}{|c|}{2002 Population $^{*}$} \\
\hline & Holotype & $\begin{array}{c}\text { Female } \\
\text { paratypes }\end{array}$ & $\mathrm{CV}$ & $\begin{array}{c}\text { Male } \\
\text { paratypes }\end{array}$ & $\mathrm{CV}$ & $\begin{array}{c}\text { Female } \\
\text { paratypes }\end{array}$ & $\mathrm{CV}$ \\
\hline Anal body diam. & 29.1 & $\begin{array}{l}31 \pm 3 \\
(26-40)\end{array}$ & 9.8 & $\begin{array}{l}31 \pm 2.7 \\
(25.8-36)\end{array}$ & 8.5 & $\begin{array}{l}31 \pm 8.3 \\
(22-50)\end{array}$ & 27 \\
\hline Rectum length & 50 & $\begin{array}{c}49 \pm 4.2 \\
(42-56)\end{array}$ & 8.6 & & & $\begin{array}{l}47 \pm 6 \\
(38-56)\end{array}$ & 13 \\
\hline Rectum/Anal body diam. & 1.7 & $\begin{array}{l}1.6 \pm 0.2 \\
(1.2-2.1)\end{array}$ & 12.8 & & & $\begin{array}{c}1.6 \pm 0.3 \\
(1.1-2)\end{array}$ & 17 \\
\hline Tail/Rectum & 6 & $\begin{array}{l}6.3 \pm 0.5 \\
(5.5-7.3)\end{array}$ & 7.7 & & & & \\
\hline Gonad length & 1250 & $\begin{array}{c}1370 \pm 187 \\
(1012-1700)\end{array}$ & 13.6 & $\begin{array}{l}1049 \pm 110 \\
(775-1206)\end{array}$ & 10.5 & $\begin{array}{l}1251 \pm 327 \\
(768-1635)\end{array}$ & 26 \\
\hline Vulva position & 1625 & $\begin{array}{c}1768 \pm 179 \\
(1375-2062)\end{array}$ & 10.1 & & & $\begin{array}{l}1620 \pm 335 \\
(1104-2070)\end{array}$ & 21 \\
\hline Vulva body diam. & 46.6 & $\begin{array}{l}57 \pm 17.8 \\
(42-107)\end{array}$ & 31 & & & $\begin{array}{c}46 \pm 8.7 \\
(33-59)\end{array}$ & 19 \\
\hline Vagina length & 26.6 & $\begin{array}{c}29 \pm 3.2 \\
(25-35)\end{array}$ & 11.2 & & & $\begin{array}{l}26 \pm 4 \\
(21-34)\end{array}$ & 15 \\
\hline Vulva-Anus distance & 207 & $\begin{array}{c}240 \pm 73.7 \\
(120-439)\end{array}$ & 30.6 & & & $\begin{array}{c}175 \pm 56.5 \\
(102-270)\end{array}$ & 32.2 \\
\hline Vulva-Anus/Tail & 0.7 & $\begin{array}{l}0.8 \pm 0.2 \\
(0.4-1.4)\end{array}$ & 28.6 & & & $\begin{array}{l}0.6 \pm 0.2 \\
(0.3-0.8)\end{array}$ & 35 \\
\hline Postvulval sac length & 121 & $\begin{array}{c}126 \pm 10.6 \\
(103-149)\end{array}$ & 8.4 & & & $\begin{array}{l}115 \pm 15.4 \\
(93-141)\end{array}$ & 13 \\
\hline $\begin{array}{l}\text { Postvulval sac length/ } \\
\text { vulva body diam. }\end{array}$ & 2.6 & $\begin{array}{l}2.3 \pm 0.5 \\
(1.2-3)\end{array}$ & 20.2 & & & $\begin{array}{c}2.5 \pm 0.3 \\
(2-2.9)\end{array}$ & 12 \\
\hline Postvulval stalk length & 29 & $\begin{array}{c}34 \pm 5.3 \\
(26-44)\end{array}$ & 15.3 & & & $\begin{array}{c}34 \pm 6.4 \\
(23-40)\end{array}$ & 19 \\
\hline Postvulval pouch length & 91 & $\begin{array}{c}91 \pm 11.2 \\
(75-120)\end{array}$ & 12.3 & & & $\begin{array}{l}81 \pm 11 \\
(70-101)\end{array}$ & 14 \\
\hline Vaginal cell cluster length & 20 & $\begin{array}{c}17 \pm 2.2 \\
(15-21)\end{array}$ & 12.6 & & & $\begin{array}{c}15 \pm 2.6 \\
(9-17)\end{array}$ & 18 \\
\hline Vaginal cell cluster diam. & 10.8 & $\begin{array}{l}13 \pm 2 \\
(10-18)\end{array}$ & 16.1 & & & $\begin{array}{c}9 \pm 2.4 \\
(6-12)\end{array}$ & 25 \\
\hline No. of eggs in uterus & & $\begin{array}{c}20 \pm 10 \\
(4-34)\end{array}$ & 49.1 & & & $\begin{array}{c}20 \pm 8.4 \\
(8-31)\end{array}$ & 42 \\
\hline $\mathrm{V}$ & 76 & $\begin{array}{c}76.4 \pm 2.1 \\
(72-81)\end{array}$ & 2.7 & & & $\begin{array}{c}76.3 \pm 3 \\
(72-81)\end{array}$ & 4 \\
\hline Gonad (as \% L) & 58.5 & $\begin{array}{c}59 \pm 2.8 \\
(55-64)\end{array}$ & 4.7 & & & $\begin{array}{l}58.4 \pm 5.5 \\
(51-66)\end{array}$ & 9.5 \\
\hline $\begin{array}{l}\text { Vagina (as \% Vulva } \\
\text { body diam.) }\end{array}$ & 57 & $\begin{array}{c}53.1 \pm 14.1 \\
(24-74)\end{array}$ & 26.5 & & & $\begin{array}{c}59 \pm 13.2 \\
(40-76)\end{array}$ & 22 \\
\hline $\mathrm{T}$ & & & & $\begin{array}{l}62.1 \pm 4.5 \\
(52-74)\end{array}$ & 7.3 & & \\
\hline Spicule length & & & & $\begin{array}{c}57 \pm 3.8 \\
(50-62)\end{array}$ & 6.7 & & \\
\hline Spicule/Anal body diam. & & & & $\begin{array}{l}1.8 \pm 0.2 \\
(1.5-2.1)\end{array}$ & 11.8 & & \\
\hline Gubernaculum length & & & & $\begin{array}{c}24 \pm 2.2 \\
(20-28)\end{array}$ & 8.9 & & \\
\hline
\end{tabular}


Table 1. (Continued).

\begin{tabular}{|c|c|c|c|c|c|c|c|}
\hline & \multicolumn{5}{|c|}{1999 Population (Type) } & \multicolumn{2}{|c|}{2002 Population ${ }^{*}$} \\
\hline & Holotype & $\begin{array}{c}\text { Female } \\
\text { paratypes }\end{array}$ & $\mathrm{CV}$ & $\begin{array}{c}\text { Male } \\
\text { paratypes }\end{array}$ & $\mathrm{CV}$ & $\begin{array}{l}\text { Female } \\
\text { paratypes }\end{array}$ & $\mathrm{CV}$ \\
\hline Gubernaculum/Spicule & & & & $\begin{array}{c}0.4 \pm 0 \\
(0.4-0.5)\end{array}$ & 8.9 & & \\
\hline Spicate tail portion & & & & $\begin{array}{c}97.2 \pm 8.4 \\
(85-117)\end{array}$ & 8.4 & & \\
\hline Spicate tail/Tail length & & & & $\begin{array}{c}0.5 \pm 0.1 \\
(0.4-0.7)\end{array}$ & 14.1 & & \\
\hline
\end{tabular}

* source of specimens for DNA extraction; males were not recovered.

and stegostom in ratio $1: 1: 2$. Cheilostom without distinct sclerotisations. Stegostom divided into two parts in sectioned specimens observed with SEM. Posterior part apparently with seven denticles or digitate projections $c a$ $2 \mu \mathrm{m}$ long, reclining against, and posteriorly connected to, stoma wall. Projections as observed with SEM in two specimens and consistent with light microscope observations on three females positioned as follows: three in dorsal sector and two in each subventral sector (the second projection in the right subventral sector was presumably sliced through in Fig. 4B). Dorsal gland opening at base of these projections. Basal lamina visible between gymnostom and stegostom (arrows in Fig. 4C). Neck region forming one fifth to one seventh of body. Pharynx divided into a cylindrical corpus comprising on average $68 \%$ of neck length, a short isthmus and an oval bulbus with transverse valves. Cardia well developed. Nerve ring at transition of corpus-isthmus. Excretory pore 15-80 $\mu \mathrm{m}$ anterior to nerve ring, wide but often difficult to find because of its lack of sclerotisation. Deirid not found. Intestine with wide lumen demarcated by refractive lining, microvilli visible anterior to rectum. Rectum long, with large sphincter cells at its beginning. Reproductive system monodelphic, prodelphic, with posteriorly reflexed ovary. Oviduct always with two distinct nuclei, located at flexure between ovary and uterus. Two opposing and offset spermatheca-like pouches present at junction of oviduct and uterus. Each pouch with one distinct terminal cell, its lining with a membranous appearance. Granulated sperm cells very large (15-34 $\mu \mathrm{m}$ long) and often compressed into serial clusters erratically dispersed throughout uterus, often alternating with developing eggs. Vulva a transverse cleft with overhanging anterior vulval lip located at $75 \%$ of body length. Vagina with thickened walls and surrounded by strong muscles. Prominent postvulval sac with anterior stalk and posterior pouch. Transition vaginapostvulval sac guarded by a cluster of four cells consisting of two pairs lying side by side. Anus a broad, curved slit in ventral view. Tail elongate-conical with fine tip. Irregular pattern of lines substituting annulation over a short distance at middle of tail, as seen by SEM. No phasmids or phasmid-like structures observed with SEM or light microscope.

Male

General morphology similar to female. Body 1.3$1.9 \mathrm{~mm}$ long, arcuate ventrad and especially so in posterior region, with tail usually curved into 1.5 coils. $\mathrm{Cu}-$ ticle finely annulated, $0.8 \mu \mathrm{m}$ wide at midbody. Lateral field indistinct, with several discontinuous lines, extending slightly posterior to cloacal opening, $3 \mu \mathrm{m}$ wide at midbody. Neck region comprising $20-25 \%$ of body length. Excretory pore from $80 \mu \mathrm{m}$ anterior to nerve ring to $6 \mu \mathrm{m}$ posterior to nerve ring. Reproductive tract occupying $50-75 \%$ of body length, monorchic, dextral, testis ventrally reflexed for 36-120 $\mu \mathrm{m}$. Vesicula seminalis containing large granulated spermatocytes. Spicules massive, arcuate, each with complex angular manubrium and well developed velum. Demarcation of shaft and velum strongly sclerotised, spicule tip not divided. Gubernaculum with ventral triangular extension and prominent crura which may protrude from cloacal aperture. Papilla formula: $1+1+1 / 1+2+1$. Seven precloacal papillae: first pair subventral on cuticular sockets at about two anal body diam. anterior to cloaca, second pair lateral, less than one anal body diam. before cloaca, third pair subventral on cuticular sockets at level of precloacal lip and one medioventral papilla located on higher socket at same level as second precloacal pair. Four papilla pairs on tail: first and fourth pair lateral, situated respectively 
at $c a$ one and two anal body diam. posterior to cloaca. Second and third papilla pair close to each other subventrally with second pair more pronounced. Tail elongateconical, narrowing sharply at midpoint and with fine tail tip. No phasmids observed with SEM or light microscope.

\section{TYPE LOCALITY}

Pitchers of Nepenthes mirabilis (Lour.) Druce in TungKai Botanical Garden, Trang Province, Southern Thailand.

\section{TYPE MATERIAL}

Holotype female (slide MDNC 4021), two paratype females (slides MDNC 4022, 4023) and four paratype males (slides MDNC 4024, 4025) deposited in the nematode collection of the Zoology Museum, Department of Biology, Ghent University, Belgium; four paratype females and four paratype males on slides with UCR-NC Accession No. 30059-30063 in the nematode collection of the Department of Nematology, University of California, Riverside, USA; three paratype females and two paratype males on UCNC slide numbers 5234-5237 in the nematode collection of the Department of Nematology, University of California, Davis, USA; two paratype females and two paratype males in the nematode type collection of CABI Bioscience, Egham, UK.

\section{DIAGNOSIS AND RELATIONSHIPS}

Baujardia mirabilis gen. n., sp. n. differs from all known nematodes in having two opposing and offset spermatheca-like pouches at the junction of oviduct and uterus. It also differs from nearly all known Rhabditida in having four cephalic setae instead of papillae, in the presence of six liplets, and in the apparent absence of phasmids.

This new genus resembles Panagrellus Thorne, 1938 in the long body size, in the elongate-conical tail and in the monodelphic, prodelphic female reproductive system with posteriorly placed vulva, thickened vaginal walls and prominent postvulval sac. However, Baujardia gen. n. differs from Panagrellus in the characters mentioned above, in the comparatively longer stegostom, and in the more robust male spicules with complex angular manubrium and undivided tip.

The liplets, cephalic setae and lack of phasmids are characters reminiscent of the genus Teratocephalus de
Man, 1876 in the family Teratocephalidae, but our new genus differs from this family in numerous respects, e.g., the amphidial apertures are located on the lips, there is a distinct differentiation between the pharyngeal corpus and isthmus and the female has an elongate reproductive system. Another taxon in which liplets and setae occur on the lip region is the family Chambersiellidae. However, members of this family have distinct labial setae, more posteriorly located amphids, a much narrower stegostom and the tail is usually equipped with a hook-like mucro. Overall, the mixture of characters resembling members of different families precludes unequivocal placement of the new genus on morphological grounds alone. In view of the molecular analysis discussed below, we conclude that it belongs in the infraorder Panagrolaimomorpha, superfamily Panagrolaimoidea and family Panagrolaimidae. The morphological diagnosis of Panagrolaimidae must be revised significantly to accommodate our new genus and the new information on some other panagrolaims recently described by Stock et al. (2002). As a result, very few unequivocal diagnostic characters currently remain for Panagrolaimidae, especially when compared to families Cephalobidae, Chambersiellidae or Brevibuccidae (we consider the latter two to be incertae sedis - see De Ley \& Blaxter, 2002). Molecular data are clearly essential as an additional source of diagnostic characters in these nematodes.

\section{MOLECULAR PHYLOGENY}

The nearly complete SSU sequences of the 22 included taxa consisted of 1491 (Rhabditophanes sp.) to 1611 (Panagrolaimus sp.) bp, with an intermediate value for Baujardia mirabilis gen. n., sp. n. (1587 bp). The secondary structure alignment comprised 1769 positions, of which 971 (55\%) were variable including $715(40 \%)$ parsimony-informative characters. The $\mathrm{A}+\mathrm{T}$ content of Baujardia gen. n. was $53 \%$.

Table 2 summarises the bootstrap support for, and monophyly of, clades in the obtained trees. NJ, MP, and ML trees consistently support (100\% bootstrap) a sister taxon relationship between the new genus and Panagrellus redivivus (L., 1767) Goodey, 1945 ( $c f$ clade A, Figs 58). Panagrolaimus sp. has an uncertain position relative to these two genera: it is placed within the same clade in the NJ and ML trees, while the MP trees place it closer to the strongly supported sister taxa Halicephalobus gingivalis (Stefanski, 1954) Andrássy, 1984 and Turbatrix aceti (Müller, 1783) Peters, 1927 (clade B). Consistent maximal support was also given to sister taxa Plectonchus 
Table 2. Monophyly $(\checkmark=$ yes, $X=$ no) and bootstrap support (\%) for clades.

\begin{tabular}{|c|c|c|c|c|c|}
\hline Clades & $\begin{array}{l}\text { NJ } \\
\text { (Fig. 5) }\end{array}$ & $\begin{array}{l}\text { MP1 } \\
\text { (Fig. 6) }\end{array}$ & $\begin{array}{l}\text { MP2 } \\
\text { (Fig. 7) }\end{array}$ & $\begin{array}{l}\text { MP majority } \\
\text { rule (Fig. 6) }\end{array}$ & $\begin{array}{c}\text { ML } \\
\text { (Fig. 8) }\end{array}$ \\
\hline \multicolumn{6}{|l|}{ Clade A } \\
\hline $\begin{array}{l}\text { (Panagrolaimus, (Baujardia (Panagrellus redivivus } \\
\text { AF036599, Panagrellus redivivus AF083007)) }\end{array}$ & 57 & $\mathrm{X}$ & $\mathrm{X}$ & 52 & $\checkmark$ \\
\hline $\begin{array}{l}\text { (Baujardia (Panagrellus redivivus AF036599, } \\
\text { Panagrellus redivivus AF083007)) }\end{array}$ & 100 & $\checkmark$ & $\checkmark$ & 100 & $\checkmark$ \\
\hline $\begin{array}{l}\text { (Panagrolaimus sp. (Halicephalobus gingivalis, } \\
\text { Turbatrixaceti)) }\end{array}$ & $\mathrm{X}$ & $\checkmark$ & $\checkmark$ & 52 & $\mathrm{X}$ \\
\hline \multicolumn{6}{|l|}{ Clade B } \\
\hline \multicolumn{6}{|l|}{ Clade C } \\
\hline (Plectonchus hunti, Panagrobelus stammeri) & 100 & $\checkmark$ & $\checkmark$ & 100 & $\checkmark$ \\
\hline (Clade C (Clade A, Clade B) $)(=$ Panagrolaimidae $)$ & 100 & $\checkmark$ & $\checkmark$ & 100 & $\checkmark$ \\
\hline $\begin{array}{l}\text { Monophyly of Strongyloidoidea (Steinernema } \\
\text { carpocapsae (Rhabditophanes, Strongyloides stercolaris)) }\end{array}$ & $\mathrm{X}$ & $\checkmark$ & $\mathrm{X}$ & 51 & $\checkmark$ \\
\hline $\begin{array}{l}\text { Monophyly of Panagrolaimoidea and Strongyloidoidea } \\
\quad\left(=\text { Infraorder Panagrolaimomorpha }{ }^{1)}\right)\end{array}$ & $\mathrm{X}$ & $\checkmark$ & $\begin{array}{l}\checkmark \text { except } \\
\text { Steinernema }\end{array}$ & $\mathrm{X}$ & $\checkmark$ \\
\hline $\begin{array}{l}\text { Rhabditomorpha }{ }^{1)}(=\text { Rhabditina }) \text { as sister taxon to } \\
\text { Panagrolaimomorpha }{ }^{1)}\end{array}$ & $\checkmark$ & & $\checkmark$ & & \\
\hline $\begin{array}{l}\text { Panagrolaimomorpha } a^{1)} \text { as sister } \\
\quad \text { taxon to Cephalobomorpha }{ }^{1)}, \text { Tylenchomorpha }{ }^{1)}\end{array}$ & & $\checkmark$ & & & $\checkmark$ \\
\hline
\end{tabular}

${ }^{1)}$ De Ley and Blaxter (2002).

hunti Stock, De Ley, De Ley, Mundo-Ocampo, Baldwin \& Nadler, 2002 and Panagrobelus stammeri Rühm, 1956 (clade C); and to a basal position for this clade within Panagrolaimidae. In other respects, our results are comparable to the SSU rDNA sequence analysis of Félix et al. (2000), for example the position of Brevibucca within Rhabditida is uncertain, variously assuming a position at the base of either infraorder Cephalobomorpha, Panagrolaimomorpha or Rhabditomorpha (De Ley \& Blaxter, 2002). Steinernema carpocapsae (Weiser, 1955) Wouts, Mráček, Gerdin \& Bedding, 1982 is another nematode whose position is unclear, both in our trees and those of Félix et al. (2000), but analysis with a more focused dataset confirms its placement in a monophyletic superfamily Strongyloidoidea (Dorris et al., 2002). As the purpose of our study was to explore phylogenetic affinities between Baujardia gen. n. and its closest relatives, we limited the number of sequences included in our dataset and it is therefore not surprising that resolution of relationships among higher taxa remains unclear in our trees (e.g. aphelenchs are not found to be monophyletic).

\section{BIOLOGY}

We assume that the pitchers of Nepenthes mirabilis are the natural habitat of Baujardia mirabilis gen. n., sp. n., considering that the species was found alive in several pitchers. These pitchers contained a relatively diverse fauna, dominated by mosquito larvae, midges and the new genus, in addition to a few dipteran and coleopteran larvae. The most abundant prey insects in the pitchers were ants and diverse flying hymenopterans. Apparently, a diverse assemblage of organisms lives in association with the pitchers of this Nepenthes species. This may represent an ecologically complex community similar to the better-known association inhabiting Sarracenia pitchers (e.g., Kitching, 2001; Greeney, 2001). Possibly the nematodes have a phoretic relationship with one or more insect species. Closely related genera are also capable of withstanding harsh environments; Turbatrix aceti (the vinegar eelworm), Panagrellus redivivus, $P$. silusiae and $P$. nepenthicola inhabit vinegar, bookbinder's paste, beer filters and pitcher plants, respectively.

Not much is known about the occurrence of nematodes in pitcher plants and they were not even mentioned in 


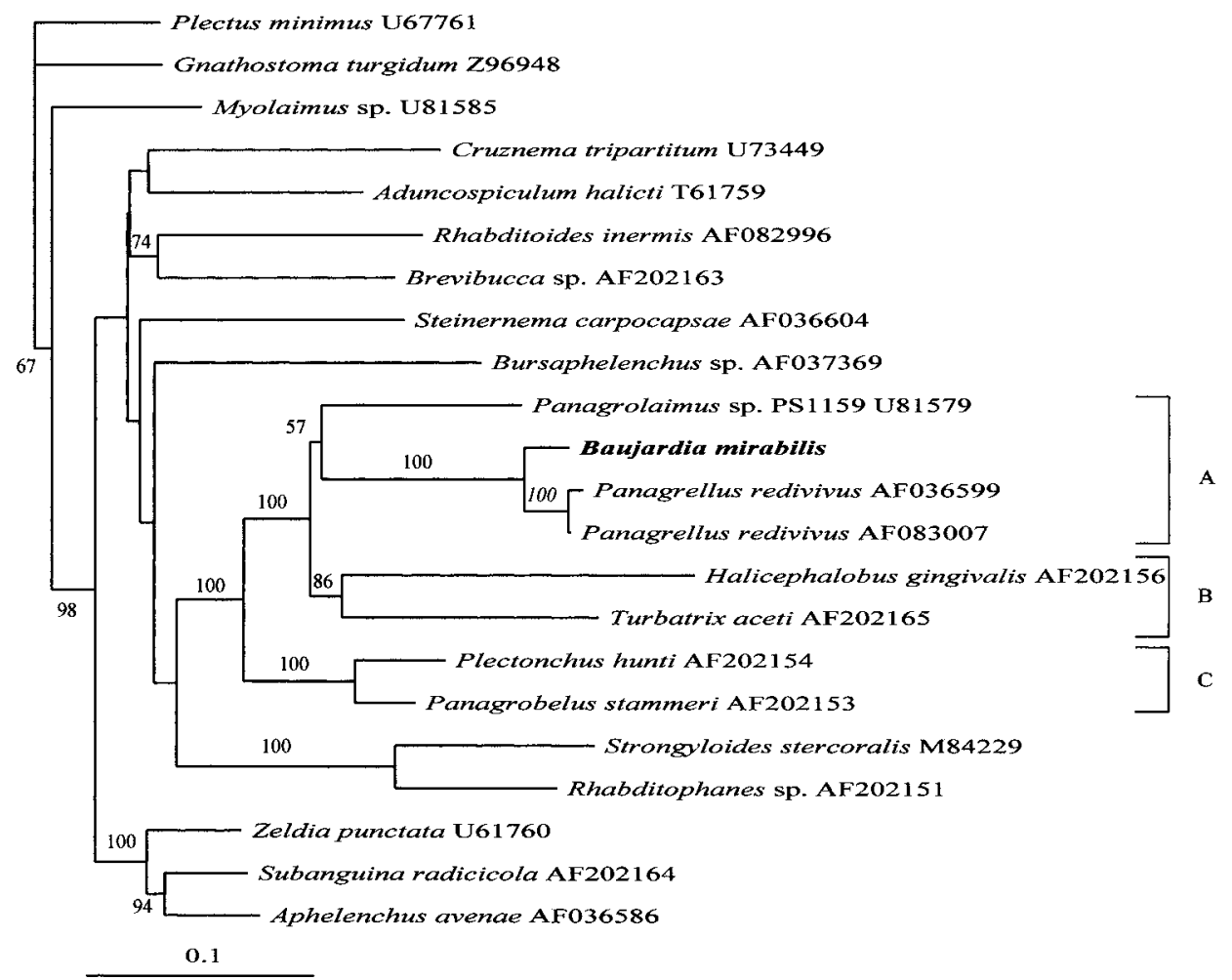

Fig. 5. Neighbour joining tree based on secondary structure alignment of small subunit rDNA. Plectus was designated as outgroup; numbers next to branches are percentage bootstrap support values from corresponding $50 \%$ majority-rule consensus tree obtained with $N J$ analysis of 3000 bootstrap replicates. A, B and $C$ indicate the three obtained clades within the Panagrolaimidae.

a reference work of the Nepenthes-inhabiting fauna by Thienemann (1933). More extensive study is needed to investigate the life cycle and biochemistry of this unusual new genus. Moreover, pitcher plants and other epiphytes are clearly a promising source of surprising new nematode taxa.

\section{Discussion}

The nematofauna of pitcher plants in Indonesia was studied by Menzel (1922), who reported the genera Plectus, Dorylaimus, Rhabditis and Diplogaster. He very briefly described a new species; Anguillula nepenthicola Menzel, 1922 which he considered as the only true inhabitant of pitcher plants and not an accidental recovery. Anguillula nepenthicola, together with several other species, including Panagrellus pycnus Thorne, 1938, was later placed in the newly erected genus Turbator by Goodey
(1943), which later (Goodey, 1945) became a junior synonym of Panagrellus.

As $P$. nepenthicola was described from the same habitat and similar geographical region as our new genus, we have briefly examined Menzel's (1922) very limited description to justify proposal of a new species for our material, especially in the absence of original illustrations for $P$. nepenthicola. Both species are comparable in length (1.8-2.7 vs 2.3-2.9 $\mathrm{mm}$ for $P$. nepenthicola) and in vulva position (average of $76 \mathrm{vs} 77 \%$ for $P$. nepenthicola). The relative tail length is, however, clearly longer in our nematodes, being on average $125 \%$ of the vulva-anus distance $v s$ only half the vulva-anus distance length in $P$. nepenthicola; the male tail is on average twice the spicula length $v s$ three times the spicula length in P. nepenthicola; the female $c$ value is 6.2-8.9 vs 9-10 in P. nepenthicola and the male $c$ value is 6.7-11 vs 12-15 in P. nepenthicola. The identity of $P$. nepenthicola became somewhat clearer with the redescription by Micoletzky and Menzel (1928) although only the male tail was illustrated. The most ap- 


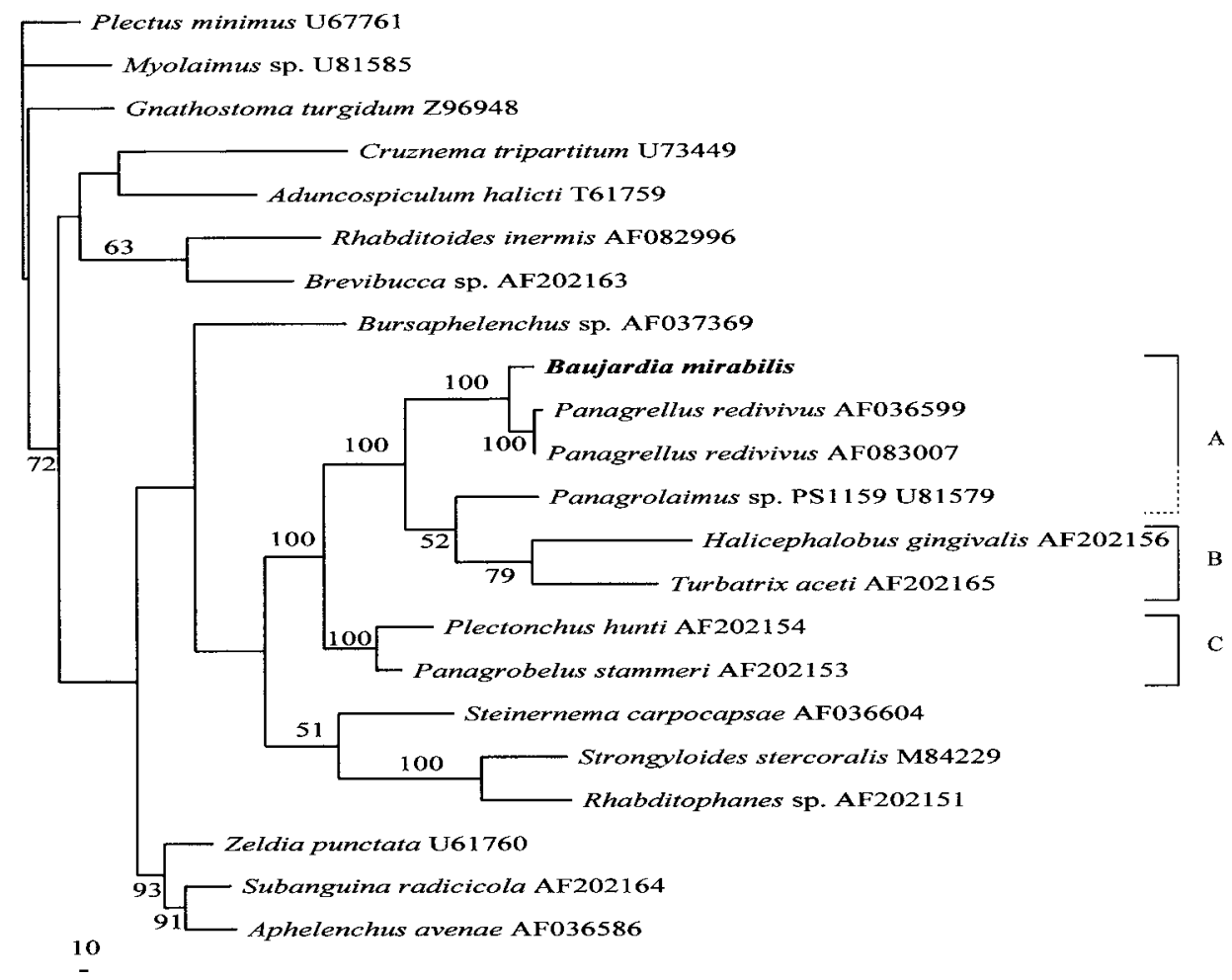

Fig. 6. First of two best trees obtained with Maximum Parsimony analysis based on secondary structure alignment of small subunit rDNA. Plectus was designated as outgroup; numbers next to branches are bootstrap values from corresponding $50 \%$ majority-rule consensus tree obtained with MP analysis of 3000 bootstrap replicates. A, B and C indicate the three obtained clades within the Panagrolaimidae. Broken line in the bracket for clade A denotes paraphyletic position of Panagrolaimus in this tree compared to the others (Figs 5, 8).

parent similarities of the new genus with the redescribed $P$. nepenthicola are the muscular vagina and long postvulval sac (103-149vs 78-150 for P. nepenthicola). However, the spicules of our new genus and species have more angular manubria and their tips are not bifid (an indistinct bifid tip can be observed on the original illustration of $P$. nepenthicola). Furthermore, the male tail is more strongly curved compared to $P$. nepenthicola and the number and positioning of the genital papillae is different. The difference in tail curvature, however, may be due to cold fixation of our nematodes.

The genital system of the new genus is panagrolaimid and shows similarities with the morphology of the female gonad of Panagrellus redivivus (according to results obtained after extraction of the genital system by Geraert et al., 1980). As in $P$. redivivus, the flexure of the genital system is formed by the oviduct and a pair of distinct oviduct cells are observed at the spermatheca side.
However, the two opposing and offset spermatheca-like pouches observed here represent a unique feature amongst described nematodes. These pouches are probably glandular, rather than having a function in sperm storage, as suggested by the unusually large size of the sperm cells and their occurrence throughout the uterus of gravid females. Possibly each compressed cluster of sperm originated from successive copulations, as multiple matings per female might be more frequent in a limited and liquid environment. Alternatively, the movement of eggs could cause the break-up of single clusters of sperm.

On morphological grounds alone, it was not immediately apparent in which family Baujardia gen. n. should be placed. The molecular analysis, however, robustly places the new genus as a sister taxon of Panagrellus within the Panagrolaimidae. As a matter of fact, the morphological similarities with Panagrellus, as discussed above, are relatively obvious a posteriori, but were not evident before 


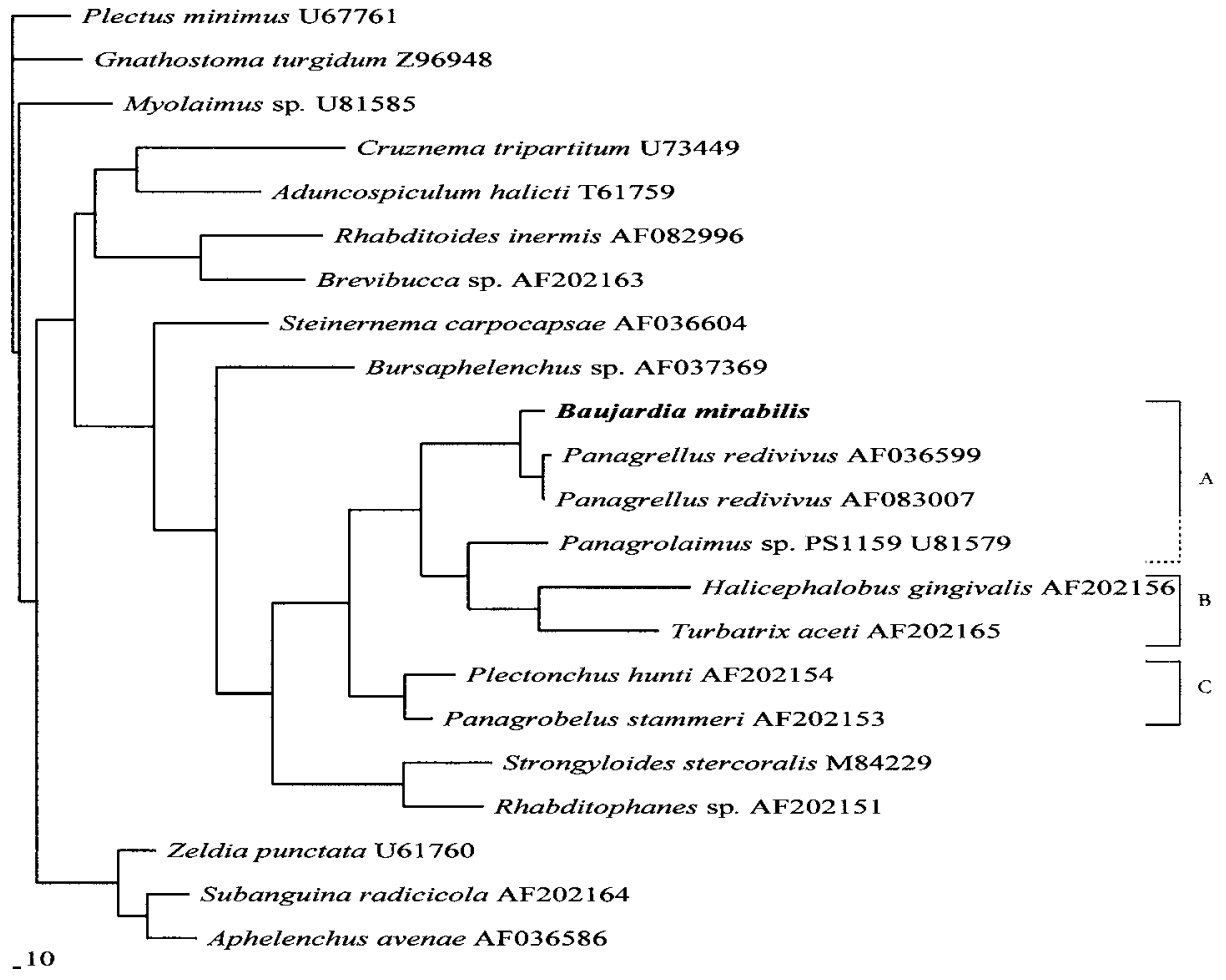

Fig. 7. Second of two best trees obtained with Maximum Parsimony analysis based on secondary structure alignment of small subunit rDNA. Plectus was designated as outgroup. A, B and $C$ indicate the three obtained clades within the Panagrolaimidae. Broken line in the bracket for clade A denotes paraphyletic position of Panagrolaimus in this tree compared to the others (Figs 5, 8).

the molecular analysis. Morphological characters such as spermatheca structure, stoma organisation, the presence of liplets, setae and amphids are generally considered to be appropriate to characterise a family. However, the placement by molecular analysis of this morphologically deviant new genus illustrates the inconsistency of these characters within the family Panagrolaimidae. As a consequence, a morphological redefinition of this family is required.

\section{Panagrolaimidae Thorne, 1937}

\section{EMENDED DIAGNOSIS}

Lip region without probolae or fimbriate processes. Labial sensilla papilliform, cephalic sensilla papilliform or rarely setiform. Cuticula finely annulated (annules usually $<1 \mu \mathrm{m}$ wide). Stoma fairly wide anteriorly, stegostom usually tapering, comprising between 50 and $66 \%$ of stoma length, never with distinct subdivisions of its lining. Pharynx with cylindrical procorpus, metacorpus cylin- drical or with valveless median bulb, terminal bulb valvate. Female reproductive system monodelphic, prodelphic. Spermatheca usually absent or axial, rarely offset as in Cephalobidae.Uterus-oviduct junction exceptionally with two offset spermatheca-like pouches. Postvulval sac present or absent. Female tail conical with pointed tip. Males without bursa, with one unpaired papilla and five to seven pairs of genital papillae, arrangement variable. Spicules often with wide velum, gubernaculum often with posteriorly expanded outline.

\section{LIST OF GENERA}

Anguilluloides Rühm, 1956

Brevistoma Mukhina, 1981

Halicephalobus Timm, 1956

= Micronema Körner, 1954

= Phytorhabditis Lordello \& De Oliveira, 1963

Panagrellus Thorne, 1938

= Anguillula de Man, 1910 pro parte

= Neocephalobus Steiner, 1936 pro parte

= Turbator Goodey, 1943 


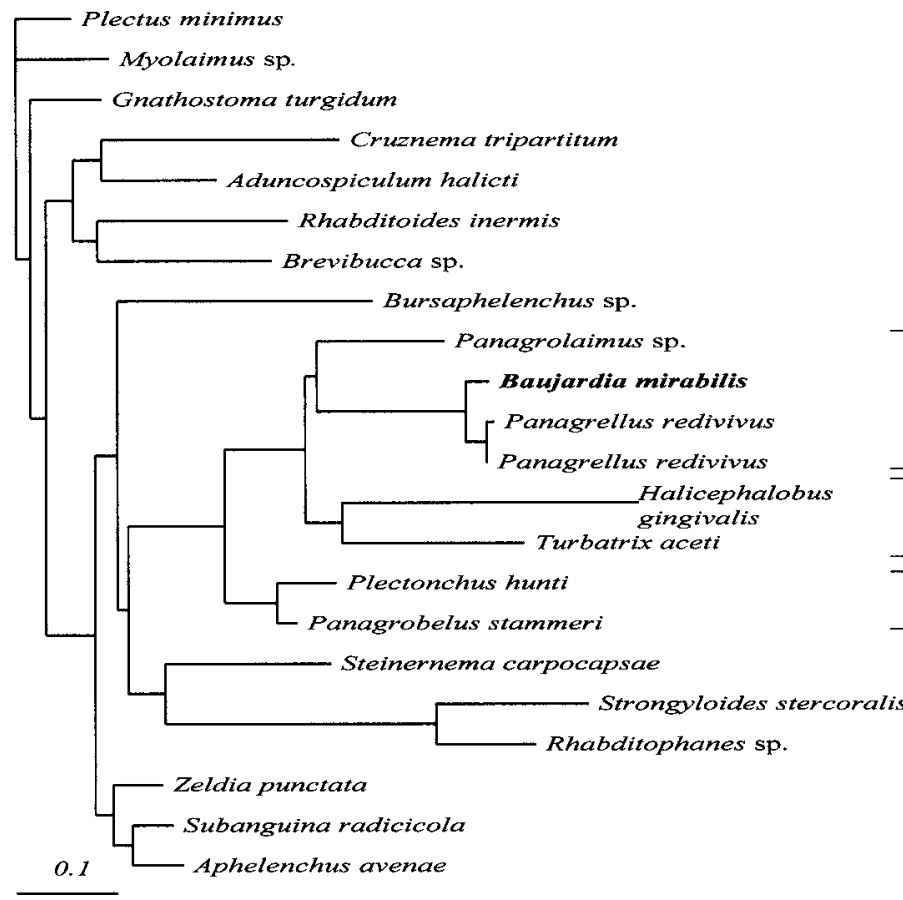

Plectidae/Plectoidea/Plectida

Myolaimidae/Myolaimoidea/Myolaimina/Rhabditida

Gnathostomatidae/Gnathostomatoidea/Spirurina/Rhabditina

Rhabditidae/Rhabitoidea/Rhabditomorpha/Rhabditina

Diplogasteridae/Diplogasteroidea/Diplogasteromorpha/Rhabditina

Rhabditidae/Rhabitoidea/Rhabditomorpha/Rhabditina/Rhabditida

Brevibuccidae/incertae sedis/Rhabditida

Aphelenchoididae/Aphelenchoidea/Tylenchomorpha/Tylenchina/Rhabditida

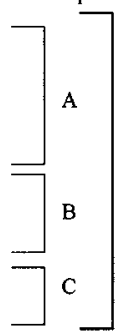

Steinemematidae/Strongyloidoidea/Panagrolaimomorpha/Tylenchina/Rhabditida

Strongyloididae/Strongyloidoidea/Panagrolaimomorpha/Tylenchina/Rhabditida

Alloionematidae/Strongyloidoidea/Panagrolaimomorpha/Tylenchina/Rhabditida

Cephalobidae/Cephaloboidea/Cephalobomorpha/Rhabditida

Tylenchidae/Tylenchoidea/Tylenchomorpha/Tylenchina/Rhabditida

Aphelenchidae/Aphelenchoidea/Tylenchomorpha/Rhabditida

Fig. 8. Maximum Likelihood tree based on secondary structure alignment of small subunit rDNA. Plectus was designated as outgroup. $A, B$ and $C$ indicate the three obtained clades within the Panagrolaimidae. Lineages of taxa are based on proposed classification scheme by De Ley and Blaxter (2002).

= Tylorhabdus Sukul, 1971

References

Panagrobelus Thorne, 1939

Panagrobelium Andrássy, 1984

Panagrolaimus Fuchs, 1930

Plectonchus Fuchs, 1930

Procephalobus Steiner, 1934

Tricephalobus Steiner, 1936

= Pseudorhabditis Kreis, 1929

= Trilabiatus Goodey, 1963

Turbatrix Peters, 1927

\section{Acknowledgements}

The authors thank P. Pholpunthin, S. Chittapun and S. Kitichate of the Department of Biology, Prince of Songkla University, Hat Yai, Thailand for organising field trips and for providing the identification of the Nepenthes species. We thank J. Vanfleteren and A. Vierstraete for the DNA sequencing and alignment. The support of $\mathrm{G}$. Borgonie in several aspects of this study is greatly appreciated. We are also grateful to M. Yoder, L. Mundo, and S. Esfahani for assistance with the production of virtual specimens.

De Ley, I.T., De Ley, P., Baldwin, J.G., MundoOCAMPO, M. \& NADLER, S.A. (1999). Three new species of Nothacrobeles (Nemata: Cephalobidae) from the Mojave Desert, California. Journal of Nematology 31, 482-497.

DE LEY, P. \& BERT, W. (2002). Video capture and editing as a tool for the storage, distribution and illustration of morphological characters of nematodes. Journal of Nematology 34 , 296-302.

De Ley, P. \& BlaXter, M.L. (2002). Systematic position and phylogeny. In: Lee, D.L. (Ed.). The biology of nematodes. London, Taylor \& Francis, pp. 1-30.

De Ley, P., Van de Velde, M.C., Mounport, D., BauJARD, P. \& CoOMANS, A. (1995). Ultrastructure of the stoma in Cephalobidae, Panagrolaimidae and Rhabditidae, with a proposal for a revised stoma terminology in Rhabditida (Nematoda). Nematologica 41, 153-182.

De Rijk, P. \& De Wachter, R. (1993). DCSE v.2.54, an interactive tool for sequence alignment and secondary structure research. Computer Applications in the Biosciences 9, 735-740 [software].

Dorris, M., Viney, M. \& Blaxter, M. (2002). Molecular phylogenetic analysis of the genus Strongyloides and related 
nematodes. International Journal for Parasitology 32, 1507 1517.

Felix, M.A., De Ley, P., Sommer, R.J., Frisse, L., Nadler, S., Thomas, K., Vanfleteren, J. \& SternBERG, P.W. (2000). Evolution of vulva development in the Cephalobina (Nematoda). Developmental Biology 221, 6886.

Geraert, E., Sudhaus, W. \& Grootaert, P. (1980). The structure of the female genital apparatus in the order Rhabditida (Nematoda). Annales de la Société Royale Zoologique de Belgique 109, 91-108.

Goodey, T. (1943). On the systematic relationships of the vinegar eelworm, Turbatrix aceti and its congeners, with a description of a new species. Journal of Helminthology 21, $1-9$.

Goodey, T. (1945). A note on the subfamily Turbatricinae and the genus Turbator Goodey, 1943. Journal of Helminthology 21, 69-70.

GreEnEy, H.F. (2001). The insects of plant-held waters: a review and bibliography. Journal of Tropical Ecology 17, 241-260.

Kishino, H. \& HAsegawa, M. (1989). Evaluation of the maximum likelihood estimate of the evolutionary tree topologies from DNA sequence data, and the branching order of the Hominoidea. Journal of Molecular Evolution 29, 170-179.

Kitching, R.L. (2001). Food webs in phytotelmata: "Bottomup" and "top-down" explanations for community structure. Annual Review of Entomology 46, 729-760.

Lockhart, P.J., Steel, M.A., Hendy, M.D. \& Penny, D. (1994). Recovering evolutionary trees under a more realistic model of sequence evolution. Molecular Biology and Evolution 11, 605-612.

MenzEL, R. (1922). Beiträge zur Kenntnis der Mikrofauna von Niederländisch Ost-Indien. II. Über den tierischen Inhalt der Kannen von Nepenthes melamphora Reinw. mit besonderer berücksichtigung der Nematoden. Treubia 3, 116-122.

Micoletzky, H. \& Menzel, R. (1922). Beiträge zur Kenntnis der mikrofauna von Niederländisch Ost-Indien. VII.
Anguillula nepenthicola Menzel aus kannen von Nepenthes gymnaphora Nees bei Tjibodas. Treubia 10, 285-290.

PAGE, R.D.M. (1996). TreeView: an application to display phylogenetic trees on personal computers. Computer Applications in the Biological Sciences 12, 357-358 [software].

PosAdA, D. \& CRAndAll, K.A. (1998). MODELTEST: testing the model of DNA substitution. Bioinformatics 14, 817-818.

SEINHORST, J.W. (1959). A rapid method for the transfer of nematodes from fixative to anhydrous glycerin. Nematologica 4, 67-69.

Stock, S.P., De Ley, P., De Ley, I., Mundo-Ocampo, M., BAldwin, J.G. \& NAdler, S.A. (2002). Panagrobelus stammeri Rühm, 1956 and Plectonchus hunti n. sp.: implications of new morphological observations for characterisation of these genera (Nematoda: Panagrolaimoidea). Nematology 4, 403-419.

SwofFord, D.L. (2002). PAUP*. Phylogenetic Analysis Using Parsimony (*and other methods), version 4.0. Sunderland, Massachusetts, USA, Sinauer Associates [software].

Tandingan De Ley, I., De Ley, P., Vierstraete, A., Karssen, G., Moens, M. \& Vanfleteren, J. (2002). Phylogenetic analyses of Meloidogyne SSU rDNA. Journal of Nematology 34, 319-327.

Thienemann, A. (1933). Die Tierwelt der Nepenthes-Kannen. Archiv für Hydrobiologie 11, 1-54.

Thorne, G. (1938). Notes on free-living and plant-parasiticnematodes. IV. (1) Panagrellus pycnus n. g., n. sp. (Cephalobidae, Panagrolaiminae). Proceedings of the Helminthological Society of Washington 5, 64-65.

Van de Peer, Y., Robbrecht, E., De Hoog, S., Caers, A., De Rijk, P. \& De Wachter, R. (1998). Database on the structure of small subunit ribosomal RNA. Nucleic Acid Research 271, 179-183.

WERGIN, W.P. (1981). Scanning electron microscopic techniques and application for use in nematology. In: Zuckerman, B.M. \& Rohde, R.A. (Eds). Plant parasitic nematodes. Volume III. New York, Academic Press, pp. 175-204. 